\title{
Some remarks on the charging capacitor problem
}

\author{
C. J. Papachristou \\ Department of Physical Sciences, Hellenic Naval Academy, Piraeus 18539, Greece \\ E-mail: papachristou@snd.edu.gr
}

\begin{abstract}
The charging capacitor is the standard textbook and classroom example for explaining the concept of the socalled Maxwell displacement current. A certain aspect of the problem, however, is often overlooked. It concerns the conditions for satisfaction of the Faraday-Henry law inside the capacitor. Expressions for the electromagnetic field are derived that properly satisfy all four of Maxwell's equations in that region.
\end{abstract}

\section{Introduction}

The charging capacitor is the standard paradigm used in intermediate-level Physics courses, textbooks and articles to demonstrate the significance of the Maxwell "displacement current" (see, e.g., [1-7]). The point is correctly made that, without this "current" term, the static Ampère's law would be incomplete with regard to explaining the conservation of charge as well as the existence of electromagnetic radiation. Also, the line integral of the magnetic field around a closed curve would be an ill-defined concept.

There is, however, a certain subtlety of the situation which is often passed by. It concerns the Faraday-Henry law both inside and outside the capacitor. The purpose of this short note is to point out the need for a more careful examination of the satisfaction of this law in the former region, i.e., in the interior of the capacitor. We will seek expressions for the electromagnetic field that properly satisfy the entire set of Maxwell's equations; in particular, the Faraday-Henry law as well as the Ampère-Maxwell law.

\section{The standard approach to the charging capacitor problem}

We consider a parallel-plate capacitor with circular plates of radius $a$, thus of area $A=\pi a^{2}$. The space in between the plates is assumed to be empty of matter. The capacitor is being charged by a time-dependent current $I(t)$ flowing in the $+z$-direction. The $z$-axis is perpendicular to the plates (the latter are therefore parallel to the $x y$-plane) and passes through their centers, as seen in Fig. 1 (by $\hat{u}_{z}$ we denote the unit vector in the $+z$ direction):

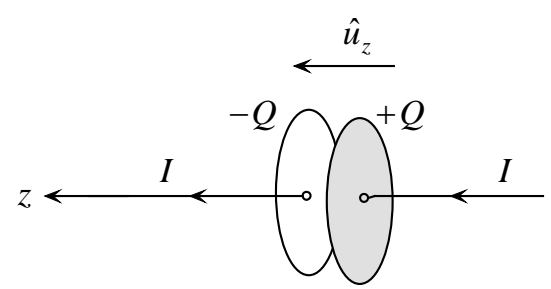

Figure 1: A current $I$ charging a parallel-plate capacitor

The capacitor is being charged at a rate $d Q / d t=I(t)$, where $+Q(t)$ is the charge on the right plate (as seen in the figure) at time $t$. If $\sigma(t)=Q(t) / \pi a^{2}=Q(t) / A$ is the surface charge density on the right plate, then the time derivative of $\sigma$ is given by

$$
\sigma^{\prime}(t)=\frac{I(t)}{A}
$$

We assume that the plate separation is very small compared to the radius $a$, so that the electromagnetic $(\mathrm{e} / \mathrm{m})$ field inside the capacitor is practically independent of $z$, although it does depend on the normal distance $\rho$ from the $z$-axis. (We will not be concerned with edge effects, thus we will restrict out attention to points that are not close to the edges of the plates.) In cylindrical coordinates $(\rho, \varphi, z)$ the $\mathrm{e} / \mathrm{m}$ field at any time $t$ will thus only depend on $\rho$ (it will not depend on the angle $\varphi$, as follows by the symmetry of the problem).

The magnetic field inside the capacitor is azimuthal, of the form

$$
\vec{B}=B(\rho, t) \hat{u}_{\varphi}
$$

A standard practice is to assume that the electric field in that area is uniform, of the form

$$
\vec{E}=\frac{\sigma(t)}{\varepsilon_{0}} \hat{u}_{z}
$$

while everywhere outside the capacitor the electric field vanishes. With this assumption the magnetic field inside the capacitor is found to be $[2,3,6]$ 


$$
\vec{B}=\frac{\mu_{0} I(t) \rho}{2 \pi a^{2}} \hat{u}_{\varphi}=\frac{\mu_{0} I(t) \rho}{2 A} \hat{u}_{\varphi}
$$

Expressions (2) and (3) must, of course, satisfy the Maxwell system of equations in empty space, which system we choose to write in the form $[1,8]$

$$
\begin{array}{ll}
\text { (a) } \vec{\nabla} \cdot \vec{E}=0 & \text { (c) } \vec{\nabla} \times \vec{E}=-\frac{\partial \vec{B}}{\partial t} \\
\text { (b) } \vec{\nabla} \cdot \vec{B}=0 & \text { (d) } \vec{\nabla} \times \vec{B}=\varepsilon_{0} \mu_{0} \frac{\partial \vec{E}}{\partial t}
\end{array}
$$

By using cylindrical coordinates and by taking (1) into account, it is not hard to show that (2) and (3) satisfy three of Eqs. (4), namely, $(a),(b)$ and $(d)$. This is not the case with the Faraday-Henry law (4c), however, since by (2) and (3) we find that

$$
\vec{\nabla} \times \vec{E}=0,
$$

while

$$
\frac{\partial \vec{B}}{\partial t}=\frac{\mu_{0} I^{\prime}(t) \rho}{2 A} \hat{u}_{\varphi} .
$$

An exception occurs if the current $I$ is constant in time, i.e., if the capacitor is being charged at a constant rate, so that $I^{\prime}(t)=0$ (this is, e.g., the assumption made in [2]). But, for a current $I(t)$ with arbitrary time dependence, the pair of fields (2) and (3) does not satisfy the third Maxwell equation.

\section{A more general formula for the $\mathrm{e} / \mathrm{m}$ field inside the capacitor}

To remedy the situation and restore the validity of the full set of Maxwell's equations in the interior of the capacitor, we must somehow correct the expressions (2) and (3) for the $\mathrm{e} / \mathrm{m}$ field. To this end, we make use of the following Ansatz:

$$
\begin{aligned}
& \vec{E}=\left(\frac{\sigma(t)}{\varepsilon_{0}}+f(\rho, t)\right) \hat{u}_{z}, \\
& \vec{B}=\left(\frac{\mu_{0} I(t) \rho}{2 A}+g(\rho, t)\right) \hat{u}_{\varphi} \\
& \sigma^{\prime}(t)=I(t) / A
\end{aligned}
$$

where $f(\rho, t)$ and $g(\rho, t)$ are functions to be determined consistently with the given current function $I(t)$ and for given initial conditions. It is easy to check that the solutions (5) automatically satisfy the first two Maxwell equations (4a) and (4b). By the Faraday-Henry law (4c) and the Ampère-Maxwell law (4d) we get the following system of partial differential equations:

$$
\begin{aligned}
& \frac{\partial f}{\partial \rho}=\frac{\partial g}{\partial t}+\frac{\mu_{0} I^{\prime}(t) \rho}{2 A} \\
& \frac{\partial(\rho g)}{\partial \rho}=\varepsilon_{0} \mu_{0} \frac{\partial(\rho f)}{\partial t}
\end{aligned}
$$

Note in particular that the "classical" solution with $f(\rho, t) \equiv 0$ and $g(\rho, t) \equiv 0$ is possible only if $I^{\prime}(t)=0 \Leftrightarrow I=$ constant in time (i.e., if the capacitor is being charged at a constant rate), as mentioned earlier.

As a special case, let us assume that the functions $f$ and $g$ are time-independent, i.e., $\partial f / \partial t=\partial g / \partial t=0 \Leftrightarrow f=f(\rho), g=g(\rho)$. From $(6 a)$ we get:

$$
f(\rho)=\frac{\mu_{0} I^{\prime}(t) \rho^{2}}{4 A} .
$$

This can only be valid if $I^{\prime}(t)=$ constant $\Leftrightarrow I^{\prime \prime}(t)=0$. On the other hand, $(6 b)$ yields: $\rho g=$ constant $\equiv \lambda \Leftrightarrow g(\rho)=\lambda / \rho$. In order for $g(\rho)$ to be finite for $\rho=0$, we must set $\lambda=0$, so that $g(\rho) \equiv 0$. The solution (5) for the e/m field inside the capacitor is then written:

$$
\begin{aligned}
& \vec{E}=\left(\frac{\sigma(t)}{\varepsilon_{0}}+\frac{\mu_{0} I^{\prime}(t) \rho^{2}}{4 A}\right) \hat{u}_{z}, \\
& \vec{B}=\frac{\mu_{0} I(t) \rho}{2 A} \hat{u}_{\varphi} ; \\
& I^{\prime \prime}(t)=0, \quad \sigma^{\prime}(t)=I(t) / A
\end{aligned}
$$

This formula preserves the familiar expression (3) for the magnetic field but corrects Eq. (2) for the electric field in order that the Faraday-Henry law be satisfied.

\section{Summary}

The purpose of this note was to point out the need to revisit the problem of the charging capacitor and to carefully examine the expressions for the e/m field in the interior of this system. As was noted, the standard formulas assumed for this field, tailor-made to satisfy the Ampère-Maxwell law, fail to satisfy the Faraday-Henry law except in the special case where the capacitor is being charged at a constant rate. We have derived a general expression for the $\mathrm{e} / \mathrm{m}$ field that satisfies the full set of Maxwell's equations for arbitrary charging rate of the system. This result reduces to the familiar set of equations in the case of a constant charging rate.

Analogous corrections need to be made to the standard expressions for the e/m field in the exterior of the capacitor. This will be the subject of a subsequent paper.

\section{Acknowledgement}

I thank Aristidis N. Magoulas for a number of helpful discussions on this problem. 


\section{References}

[1] D. J. Griffiths, Introduction to Electrodynamics, $3^{\text {rd }}$ Edition (Prentice-Hall, 1999).

[2] R. K. Wangsness, Electromagnetic Fields, $2^{\text {nd }}$ Edition (Wiley, 1986).

[3] A. Shadowitz, The Electromagnetic Field (McGraw-Hill, 1975).

[4] V. Rojansky, Electromagnetic Fields and Waves (Dover, 1979).

[5] J. D. Jackson, Maxwell's Displacement Current Revisited, Eur. J. Phys. 20 (1999) 495.

[6] K. T. McDonald, Magnetic Field in a TimeDependent Capacitor (Princeton, 2017).

[7] K. T. Selvan, A Revisiting of Scientific and Philosophical Perspectives on Maxwell's Displacement Current, IEEE Antennas and Propagation Magazine, Vol. 51, No. 3 (2009) 36.

[8] C. J. Papachristou, Introduction to Electromagnetic Theory and the Physics of Conducting Solids (META Publishing, 2017). 